\title{
Emprunts en Français Contemporain des Cités (FCC) : plusieurs décennies d'un turnover permanent
}

\begin{abstract}
Résumé
En Français Contemporain des Cités (FCC) on note l'existence d'un nombre relativement important d'emprunts, essentiellement aux langues pratiquées par des ressortissant(e)s de communautés issues de l'immigration. De tels emprunts, en grande partie apparus lors des dernières décennies, s'inscrivent dans un processus permanent de renouvellement et/ou de remplacement de lexèmes employés plus particulièrement par des locuteur(e)s des quartiers dits défavorisés. De ce fait, en synchronie dynamique, des termes autochtones et allochtones se concurrencent les uns avec les autres et un turnover permanent s'instaure entre des lexèmes empruntés et des lexèmes du français standard, circulant. Ceci témoigne de la dynamique actuelle existant entre deux positions antagonistes, à savoir une acceptation des emprunts tels quels envers une assimilation plus ou moins importante de ceux-ci.
\end{abstract}

Mots-clés : français contemporain des cités (FCC), emprunts, mot métissés, turnover lexical, verlan, verlanisation.

\section{Summary}

In Français Contemporain des Cités (Contemporary French) there is a relatively large number of loans, mainly to the languages practiced by people from the immigrant communities. Such borrowing, which has largely emerged in recent decades, is part of an ongoing process of renewing and / or replacing lexemes employed, in particular by speakers in so-called disadvantaged neighborhoods. 
Consequently, in dynamic synchronism, indigenous and non-native terms compete with each others and a permanent turnover is established between borrowed lexemes and circulating lexemes of standard French. This testifies to the current dynamic between two antagonistic positions, namely an acceptance of the borrowing as such towards a more or less important assimilation of these.

Keywords: French Contemporary Speech (FCC), borrowings, lexical turnovers, verlan,

\section{Introduction}

Depuis plusieurs décennies on constate en Français Contemporain des Cités (FCC) l'existence d'un nombre important d'emprunts, pour l'essentiel issus des langues et vernaculaires utilisés par les ressortissant(e)s de communautés issues de l'immigration demeurant dans des cités et des quartiers, qui sont autant de lieux de relégation(s) depuis de nombreuses années ${ }^{1}$. Ces emprunts, en grande partie apparus lors des dernières décennies, s'inscrivent dans un processus permanent de renouvellement et/ou de remplacement de lexèmes employés plus particulièrement par les locuteur(e)s de ces quartiers dits défavorisés ${ }^{2}$.

Ainsi, si l'on examine la série synonymique rouiller, squatter le hall, le porche, chauffer le béton, cramer le bitume, tenir le mur, on constate d'après les utilisations qui sont faites de ces termes et locutions, que l'expression tenir le mur s' inscrit aussi dans une autre série, à savoir dans l'ordre chronologique d'apparition tenir le mur - être un hétiste - être un muriste.

Tenir le mur est le calque d'une expression existant en arabe dialectal maghrébin, plus particulièrement algérien; hétiste (dans l'expression être un hétiste) est issu de la variante linguistique algéroise de l'arabe (cf. plus loin). Dans

1 Jean-Pierre Goudaillier, 2007, « Le français contemporain des cités (FCC) - Lieu de brassage linguistique ", in Mehrsprachigkeit in frankophonen Raümen (Sabine Bastian, Elisabeth Burr [Hg.]), München, Martin Meidenbauer, Coll. 'SprachKultur-Gesllschaft', Vol. 1, p. 47-62. Voir aussi Jean-Pierre Goudaillier, 2007, «Français contemporain des cités (FCC) : langue en miroir, langue du refus de la société », Adolescence, 59 (« Droit de cité »), L’Esprit du temps, p. 119-124.

2 Voir, entre autres, David Lepoutre, 1997, Cour de banlieue, codes rites et langages, Paris, Odile Jacob, ainsi que Jean-Pierre Goudaillier, 2009, « Pratiques langagières et linguistiques révélatrices des pratiques sociales de jeunes résidant en Z.U.S. ", Adolescence, 70 («Langages »), L’Esprit du temps, p. 849-857. 
être un muriste le mot allochtone d'origine arabe hétiste contenu dans être un hétiste est remplacé par le terme autochtone français mur.

Cet exemple confirme qu'en synchronie dynamique, des termes autochtones et allochtones se concurrencent les uns avec les autres et permet de constater qu'un turnover permanent s'instaure entre des lexèmes empruntés et des lexèmes du français standard, circulant. Ceci témoigne de la dynamique qui existe entre deux comportements antagonistes actuels des locuteur(e)s, à savoir une acceptation des emprunts tels quels ou une assimilation plus ou moins importante de ceux-ci.

Revenons-en à rouiller : c'est un terme fort pour rendre compte du caractère dur du quotidien, tout comme le sont les termes de la série synonymique mentionnée plus haut, qu'exprime d'une autre manière tout aussi explicite l'expression tenir le mur, qui renvoie à une pratique couramment constatée. La littérature contemporaine en témoigne aussi : «... ils nous ont vus qui tenaient le mur et tuaient le temps, les potes et moi... » (Smail 2001 : 21). Autres exemples : «Quand j'avais xlas de leurs petits plans pourraves : tuer le temps dans les caves, tenir le mur avec leurs épaules, chourrer un caddie chez Auchan... » (Smaill 2001 : 29) ; «... éviter ainsi de me faire tailler grave par la caille qui tient le mur des couloirs au niveau -3 et donne une note et son avis sur tout pote qui passe leuss, son allure, son attitude...» (Smaïl, $2001: 38$ ).

Si la précarité est particulièrement importante, ce n'est pas seulement le mur, qui est symboliquement tenu, mais tout le bâtiment et la culture d'origine maghrébine va même préciser les choses : une blague, qui consiste à dire que les hommes tiennent le bâtiment par l'extérieur pendant que les femmes le font par l'intérieur, est entendue entre les tours et les barres. C'est cette même origine culturelle, qui nous vaut le lexème hétiste cité plus haut : « ... des hétistes, y’en a aussi ici, dans cette cité... comme partout, toujours la même galère... c'est la crise » (Goudaillier, CARGO [95-96])3. Hétiste est formé à partir du mot, qui désigne en arabe dialectal maghrébin le mur. Il est employé à Alger même; à ce titre il fait partie du stock lexical du niveau populaire / argotique de la variante linguistique algéroise. Rappelons qu'en français contemporain des cités (FCC) hétiste a été supplanté par muriste, ce qui confirme l'existence d'un turnover lexical ${ }^{4} \mathrm{y}$ compris au sein même du vocabulaire des cités.

3 L'indication Goudaillier, CARGO $[\mathrm{xx}-\mathrm{xx}]$ renvoie aux enquêtes de terrain effectuées dans le cadre du Centre de Recherches Argotologiques (CARGO) ; les chiffres indiqués entre [ ] correspondent aux dates des enquêtes.

4 Jean-Pierre Goudaillier, 2012, «Pratiques linguistiques en français contemporain des cités (FCC) révélatrices des faits sociaux et culturels des cités françaises », in 
L'emprunt par le français à l'arabe et au berbère n'est pas un phénomène récent, rappelons-le, comme le confirme toute observation d'un dictionnaire, étymologique ou non, de la langue française. Le français contemporain des cités n'échappe pas à ce phénomène, ce qu'illustrent les termes suivants (en graphie francisée, ce qui rend compte de leur intégration dans le système linguistique du français) : arhnouch «policier », bzazel (ou bzèz(il), bzez) «sein(s)», casbah "maison", choune "sexe féminin », haram "péché », hralouf "porc», mesquin "pauvre type », seum « rage », shitan « diable», toubab «français d'origine» (> babtou + boubta par transformation de type verlanesque), zetla « haschisch", $z o u z$ « fille, femme ». Tous ces emprunts, qui ne sont pas entrés au même moment en français contemporain des cités, voire en français standard, sont en concurrence avec ceux du stock lexical du français, quel que soit le registre auquel ils appartiennent, et ceux-là peuvent dans bien des cas supplanter ceux-ci. C'est de cette manière que s'opère un véritable turnover, un renouvellement du vocabulaire en français contemporain des cités. Dans le même ordre d'idées, ce que l'on désigne par mots métissés (pour l'essentiel de type arabe + français) contribue aussi à l'apparition de nouveaux termes, qui viennent concurrencer ou remplacer d'autres termes déjà présents dans le vocabulaire employé.

Un blédard, un bledman, un blédos, un blédien sont tous des termes désignant celui qui arrive de son bled, celui qui est ignorant, le paysan (au sens de « rustre »), mais aussi la personne (plutôt d'origine maghrébine) qui arrive tout droit de son pays d'origine. La suffixation en -ien a recours à un suffixe du registre standard, à la différence des suffixations argotiques en -os, -ard de bled. On notera l'existence du synonyme bledman (bled + man) et du verlan deblé man (bled $>$ deblé + man). D'un point de vue étymologique l'arabe classique bilafid a donné en arabe maghrébin bled avec le sens de terrain, ville, pays. C’est par l'intermédiaire de l'argot militaire d'Afrique du Nord à la fin du XIX ${ }^{\mathrm{e}}$ siècle (époque de la colonisation) que ce terme est passé en argot.

Kiffer « aimer » est à rapprocher d'une part de l'arabe kiff (mélange de cannabis et de tabac) et du fait, par conséquent, d'aimer le kiff, d'autre part de la locution être kiff de qqn « aimer quelqu'un ».

Scarlette «fille, femme» est la suffixation en -ette du mot scarla, le verlan de lascar "vaurien », "gars", "gars de la cité » (avec pour ce dernier sens une connotation de ruse, de force, autant de qualités attendues de la part d'un soldat, ce qui renvoie à l'image de quelqu'un qui doit faire face aux exigences, aux dure-

Alicja Kacprzak, Agnieszka Konowska et Mieczysław Gajos (éds), Pluralité des cultures : chances ou menaces? Analyses linguistiques et didactiques, Łódź-Łask: Wydawnictwo LEKSEM, p. 133. 
tés de la vie). Il s'agit au départ du persan laskhar « soldat », qui est passé ensuite en arabe, avant d'être emprunté en français à différentes époques (cf. le sens de lascar en langue standard, différent de celui attribué à ce lexème en français contemporain des cités).

Le cas du mot toubab "français (de souche) », et de tous ceux qui en découlent est particulièrement intéressant : au Maghreb, plus particulièrement en Algérie, pendant la période coloniale les autochtones de langue arabe utilisaient le mot arabe maghrébin algérien țbib «sorcier» (en arabe țabīb «savant») pour désigner le médecin (" sorcier»), mais aussi tout homme blanc européen (cf., entre autres, Ben Smaïl 1994 : 135). Ce mot est devenu toubib « médecin » en français standard et toubab (prononcé [tubab]) en français contemporain des cités pour désigner le Français de souche ou souchien ${ }^{5}$ : « quand tu veux dire le Blanc, tu dis le çaifran ou le toubab ou le gaulois» (Décugis et Zemouri, 1995 : 173). Toubab est verlanisé en babtou et apocopé en bab, avant même d'être reverlanisé en boubta. La verlanisation de toubab en babtou et la reverlanisation en boubta ne correspondent nullement à un phénomène isolé ; on peut relever l'exemple de femme $>$ meuf (verlan 1) $>$ feumeu (verlan 2), mais aussi, parmi d'autres, ceux de mère $>$ reum (verlan 1) > meureu (verlan 2), choper $>$ peucho (verlan 1) > peuoch (verlan 2), comme ça > comme aç (verlan 1 en argot traditionnel) > ascom, askeum (verlan 2 en FCC) > asmeuk (verlan 3, à nouveau en FCC), à côté de celui de toubab (français de souche) $>$ babtou (verlan 1) $>$ boubta (verlan 2 ; permutation des voyelles et non des syllabes) ${ }^{6}$ qui vient d'être présenté. La reverlanisation participe donc amplement au turnover, dont il a été déjà fait état, car elle constitue un procédé relativement simple de production néologique. En plus des graphies toubab et babtou on note aussi l'existence de $2 b a b$ et $b a b 2$, qui utilisent le chiffre 2 à la manière anglo-américaine. La forme féminine correspondant à toubab est toubabesse (prononcé [tubabes]) : Française de souche :... "que le résident veuille cochonner une toubabesse, comme ils disent, il communique son type de donzelle au contact, s'acquitte du tarif...» (Ngoye, 1996: 169). Toubab est repris par la littérature dite 'de banlieues' : “C'est un toubab, enfin un Blanc, un camembert, une aspirine quoi...» (Guène, 2004: 97). Suit un exemple

5 Pour Français de souche on relève de nombreux synonymes en Français contemporain des cités, parmi lesquels blonblon, blondin, céanf, céfran, Chabert, de souche, fesses d'aspirine, fesses d'oignon, fils de Clovis, from, fromage blanc, gaulois, pâté-rillettes, rillette, roum, roumi.

6 Christian Bachmann et Luc Basier, 1984, "Le verlan: argot d'école ou langue des keums? ", Mots, 8, p. 169-187. Voir aussi les exemples de verlan cités dans Jean-Pierre Goudaillier, 2001, Comment tu tchatches! - Dictionnaire du français contemporain des cités, Paris, Maisonneuve et Larose (1ère édition : 1997). 
de reprise en dehors de l'univers des banlieues : « ... les babtous sont avantageuses si elles ont un studio et du fric pour nous entretenir. Comme elles sont toutes excitées de fréquenter la racaille, tout le monde s'y retrouve » (Libération, 15 avril 1994, p. 29, extrait de l'interview de Leonce, article de Nathalie Gathié, «Leçons de drague black»; dossier «Banlieue », Chronique de la Cité « Derrière les murs » à Villiers-le-Bel). Autres exemples : « les toubabs, les babs, c'est les français (Goudaillier, [CARGO 95-96]) ; « ...j’ai bien kiffé Barbès Family, Impact 91 et la leçon de hip hop donnée par les deux babtous et le re-noi du groupe $\mathrm{E}=2 \mathrm{MC}$ » (L'Affiche, $\mathrm{n}^{\circ}$ 47, juillet-août 1997, «Courrier des lecteurs », p. 7) ; « les babtous, on les appelle aussi fesses d'aspirine ou fesses d'oignon» (Goudaillier, [CARGO 2000]) ${ }^{7}$. Le rappeur Oxmo, parmi d'autres, utilise ce terme dans un de ses textes : «...les marionnettes vaudou, bounty ${ }^{8}$, disent à tout-va qu'ils sont pas comme nous / M'affichent devant les babtous..... »".

L’analyse des exemples de lexèmes présentés dans cet article, tous tirés du français contemporain des cités, permet de confirmer que dans l'univers des cités et quartiers français non seulement le recours aux emprunts aux langues parlées par les communautés issues de l'immigration, mais aussi l'utilisation par ces mêmes communautés de mots métissés (de type arabe + français pour leur plus grande partie), ainsi que le procédé de verlanisation, mais aussi et surtout celui de reverlanisation, contribuent à l'émergence et à la persistance d'un véritable turnover lexical. Ce processus dynamique est un des facteurs à la base du renouvellement constant des éléments constitutifs du vocabulaire du parler des cités.

\section{Références bibliographiques}

Bachmann Christian et Basier Luc, 1984, «Le verlan : argot d'école ou langue des keums? », Mots, 8, p. 169-187.

Ben Smail Mohamed, 1994, Dictionnaire des mots français d'origine arabe, Tunis, Ster.

7 Cf. Note 3.

8 Un Bounty est une friandise faite de noix de coco entourée de chocolat. Par effet métaphorique, c'est l'image de la noix de coco blanche entourée de chocolat noir, qui est utilisée à l'adresse d'une personne africaine ou antillaise qui pense, réagit, vit comme un de souche, un souchien (français d'origine), ou est supposée agir comme tel par les membres de sa communauté (pour des exemples d'emploi de ce terme cf. Goudaillier 2001 : 75).

9 Oxmo, Sortilège, Album Opéra Puccino», Delabel, 72438457862 6, 1998. 
Décugis Jean-Michel et Zemouri Aziz, 1995, Paroles de banlieue, Paris, Plon. Goudaillier Jean-Pierre, 2001, Comment tu tchatches! - Dictionnaire du français contemporain des cités, Paris, Maisonneuve et Larose (1ère édition : 1997). Goudaillier Jean-Pierre, 2007, « Le français contemporain des cités (FCC) - Lieu de brassage linguistique », in Sabine Bastian, Elisabeth Burr [Hg.], Mehrsprachigkeit in frankophonen Raümen, München, Martin Meidenbauer, Coll. "Sprache-Kultur-Gesellschaft", vol. 1, p. 47-62.

Goudaillier Jean-Pierre, 2007, «Français contemporain des cités (FCC) : langue en miroir, langue du refus de la société », Adolescence, 59 ("Droit de cité»), L'Esprit du temps, p. 119-124.

Goudaillier Jean-Pierre, 2009, « Pratiques langagières et linguistiques révélatrices des pratiques sociales de jeunes résidant en Z.U.S. ", Adolescence, 70 (« Langages »), L'Esprit du temps, p. 849-857.

Goudaillier Jean-Pierre, 2012, «Pratiques linguistiques en français contemporain des cités (FCC) révélatrices des faits sociaux et culturels des cités françaises », in Alicja Kacprzak, Agnieszka Konowska et Mieczysław Gajos (éd.), Pluralité des cultures: chances ou menaces? Analyses linguistiques et didactiques, Łódź-Łask: Wydawnictwo LEKSEM, p. 129-136.

Guène Faïza, 2004, Kiffe kiffe demain, Paris, Hachette Littératures.

Lepoutre David, 1997, Cour de banlieue . Codes rites et langages, Paris, Odile Jacob, 1997.

N'goye Achille F., 1996, Agence Black Bafoussa, Paris, Gallimard (Série noire, $\left.\mathrm{n}^{\mathrm{0}} 2413\right)$.

Smaïl Paul, 2001, Ali le magnifique, Paris, Denoël. 